\title{
Head motion invariance in three-dimensional video analysis system for the analysis of facial movement
}

\author{
Barbara Koneczny $^{1 *}$, Nicole M. Artner ${ }^{1}$, Eva Gyoeri ${ }^{2}$, Igor Pona ${ }^{2}$, Chieh-Han J. Tzou ${ }^{2}$ and Walter G. Kropatsch ${ }^{1}$ \\ ${ }^{1}$ Pattern Recognition and Image Processing Group, TU Wien, Vienna, Austria \\ ${ }^{2}$ Division of Plastic and Reconstructive Surgery, Department of Surgery, Medical University of Vienna, Austria
}

In the temporal analysis of facial movement an objective, quantitative analysis is desirable. This can be achieved with the help of 3D systems, like the proposed mirror setup in Figure 1, which was introduced by Frey et al. [1] in 1994. The main advantage of this mirror setup is that it introduces an inexpensive virtual multi camera system. Depending on the angle $\alpha$, the face of a patient can be seen in up to five different views (Figure 1). This system was designed to measure the progress in facial palsy patients by analyzing the $3 \mathrm{D}$ motion of interest points of in the face (e.g. left and right corner of the mouth). Unfortunately, the motion of these interest points is overlaid by the global motion of the head of the patients.

Invariance to head motion can be achieved by modelling the its motion using three static markers and introducing an object centered coordinate system. These static markers (Figure 1) are placed above bony structures so that only motion arising from the head influences their position. The introduction of an object centered coordinate system enables to distinguish between motion that arises from the head (global) and motion that arises from the facial movements (local). Based on the methodology introduced by Lin et al. [2], the head motion can be modelled in three dimensional space (Figure 2).

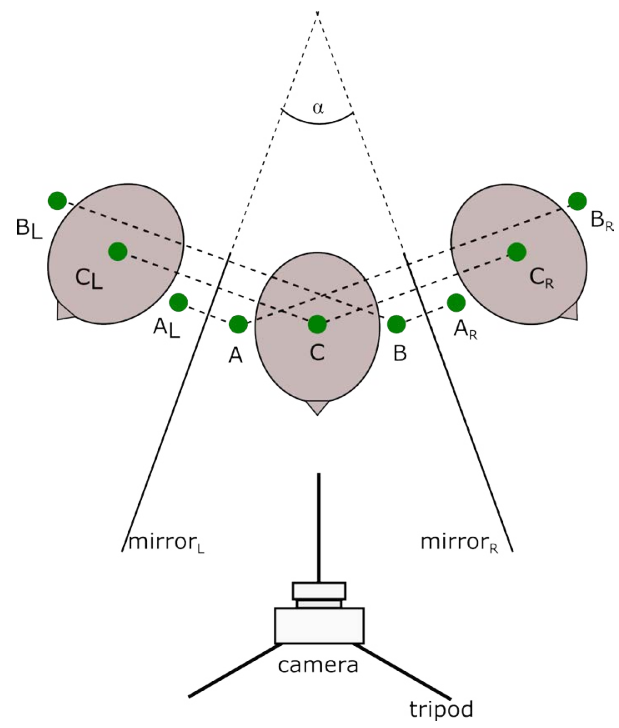

Figure 1. The mirror setup is composed of two mirrors at an arbitrary angle $\alpha$. The subject is positioned inside this setup. The points $A, B$ and $C$ are the static markerpoints in the central view. The points $A_{L}, B_{L}$ and $C_{L}$ are the virtual markerpoints in the left mirror, $A_{R}, B_{R}$ and $C_{R}$ the virtual markerpoints in the right mirror. The camera recording the scene is positioned on a tripod.
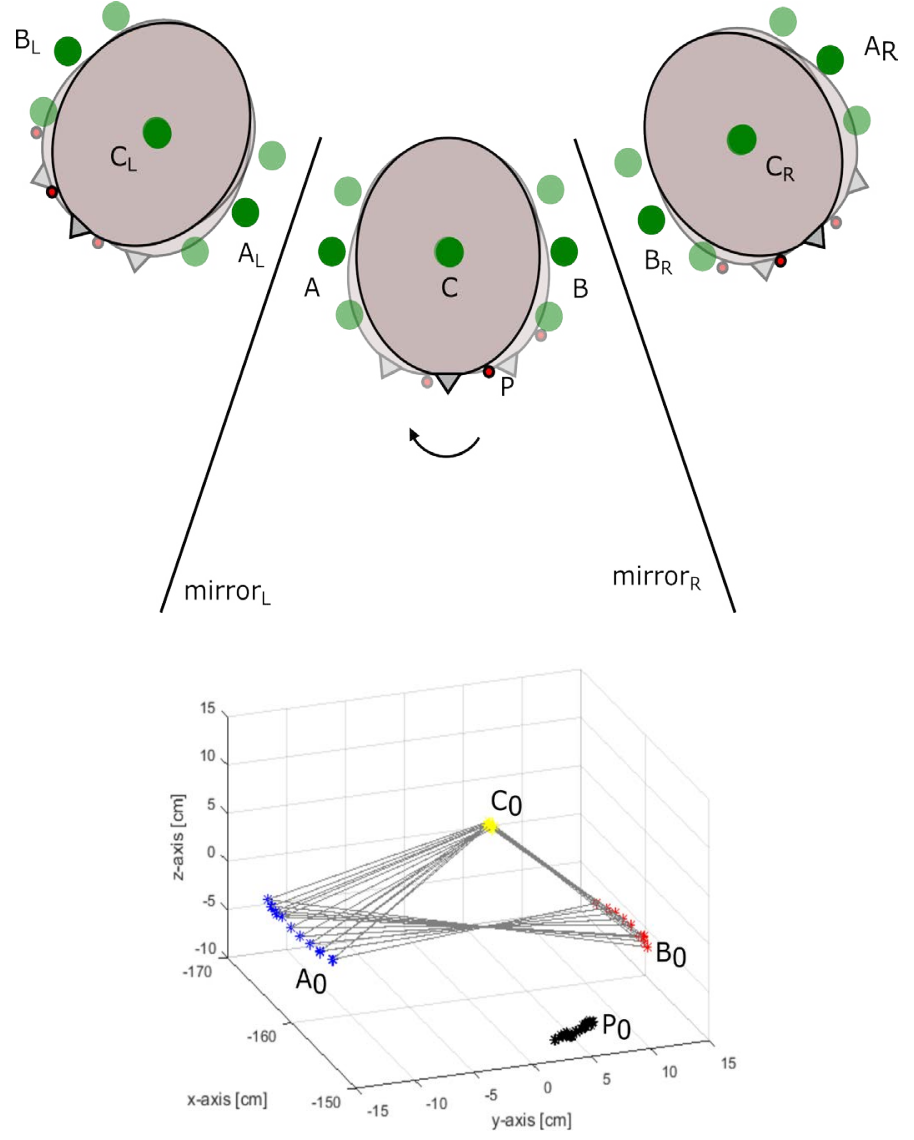

Figure 2 .(A): The experimental setup consisted of an artificial head model on a rotary plate, three static markers $(A, B$ and $C)$ and the mirror setup. $P$ is an interest point, which moves due to the rotation of the head. The artificial head model was manually rotated in $5^{\circ}$ steps in counter-clockwise direction. (B) The static markers are used to calculate the $3 \mathrm{D}$ head motion. The $3 \mathrm{D}$ positions of the markers are represented by $A_{0}, B_{0}$ and $C_{0}$. The $3 \mathrm{D}$ position of the observation point is represented by $P_{0}$.

Correspondence to: Barbara Koneczny, Pattern Recognition and Image Processing Group, TU Wien, Vienna, Austria, E-mail: koneczny@prip.tuwien. ac.at

Received: May 08, 2017; Accepted: May 25, 2017; Published: May 29, 2017 


\section{References}

1. Frey M, Jenny A, Giovanoli P, Stüssi E (1994) Development of a new documentation system for facial movements as a basis for the international registry for neuromuscular reconstruction in the face. Plast Reconstr Surg 93: 1334-1349. [Crossref]
2. Lin I-C, Yeh J-S, Ouhyoung M (2002) Extracting 3D facial animation parameters from multiview video clips. IEEE Computer Graphics and Applications 22: 72-80.

Copyright: $\odot 2017$ Koneczny B. This is an open-access article distributed under the terms of the Creative Commons Attribution License, which permits unrestricted use, distribution, and reproduction in any medium, provided the original author and source are credited. 